\title{
ATOPY, RISK FACTOR FOR OTITIS MEDIA WITH EFFUSION
}

\author{
Ileana Ioniuc, Aurica Rugina, Alina Murgu, Irina Criscov, Alice Azoicai, \\ Paula Popovici, Bogdan Stana, Monica Alexoae \\ $2^{\text {nd }}$ Clinic of Pediatrics, "Gr. T. Popa” University of Medicine and Pharmacy, Iasi
}

\begin{abstract}
Chronic otitis, one of the major causes of pediatric hypoacusia is frequent associated with some allergic diseases. The continuous rice of atopic diseases prevalence in childhood, involves a multidisciplinary approach of Eustachian tube dysfunction, adenoid inflammation or recurrent otitis, for a complex pathogenic management. The objective of this study was to determinate the association between otitis media and the allergic diseases with the implication in the treatment and evolution of this patients.

Methods. Retrospective study on 92 children, between 6 month and 6 years, admitted in II Pediatric Clinic, Clinical Hospital of Children "Sf. Maria" lasi with diagnosis of acute otitis media, for a period of 1 year.

Results. Majority of cases (61patients) have been suppurate otitis, 31 cases have been otitis media with effusion out of which 23 have been recurrent. Association with allergic diseases has been more frequent in children with serous otitis media (26/31 cases). 4 cases with suppurate otitis media have developed adverse reaction to antibiotics with major implication in the cases evolution. Association with gastroesophageal reflux was relatively similar between cases with serous otitis and the suppurate ones (10/31 cases and 11/61 cases). From the atopic patients with gastroesophageal reflux, 10 presented cow's milk protein allergy.

In conclusion, the children with otitis media, especially those with recurrent none suppurate form should be investigated for atopy and, eventually, for gastroesophageal reflux, possibly with determination of the allergic markers or of pepsin in the otic exudates, in order to a targeted therapeutic management.
\end{abstract}

Keywords: otitis, effusion, allergy, children

\section{INTRODUCTION}

Chronic otitis media with effusion represent one of the most frequent causes of hypoacusia in pediatric population, often related with allergic diseases. Increased incidence of children atopic diseases in the last decades, involves a multidisciplinary approach of adenoiditis, Eustachian tube dysfunction and otitis media, leading to a complex pathogenic treatment.

\section{OBIECTIVUL STUDIULUI}

The aim of this study is to determinate the association between otitis media with the allergic diseases and the implication of these association in the treatment and the evolution of these patients.

\section{METHODS}

We perform a retrospective study that included 92 children (6 months -6 years) hospitalized in II Pediatric Clinic for a period of one year (January
2015 - January 2016) with a diagnosis of acute otitis media. The study protocol included: data concerning the recurrent episodes of otitis media and atopic or non-atopic, personal and familial risk factors, therapeutic difficulties, investigation of infectious, allergic and malformative etiology and the association with allergic diseases. In vitro determination of total IgE was made by a of antigen - antibody agglutination reaction, using a Randox system (Randox Laboratories Limited, United Kingdom) and the determination of specific IgE - Pediatric Panel has been achieved with the EUROLINE system (Euroimmun Mediziniche Labordiagnostika GmbH, Lubeck, Germany) which provides a semi quantitative analysis for human IgE antibodies to outdoor or indoor air allergens and food allergens.

\section{RESULTS}

The study group was divided into 2 subgroups: subgroup 1 - children diagnosed with suppurated

Corresponding author:

Alina Murgu, "Gr. T. Popa”, University of Medicine and Pharmacy, 16 Universitatii Street, lasi

E-mail: m_alina_66@yahoo.com 
otitis (61 cases) and subgroup 2 - children diagnosed with serous otitis media. The distribution by area of origin, sex and age did not differ significantly between the 2 subgroups.

Atopy was more evident in subgroup $2(26 / 31$ cases) than in 1 subgroup (15/61 cases) (Fig. 1) and in patients with recurrent episodes. (Fig. 2)

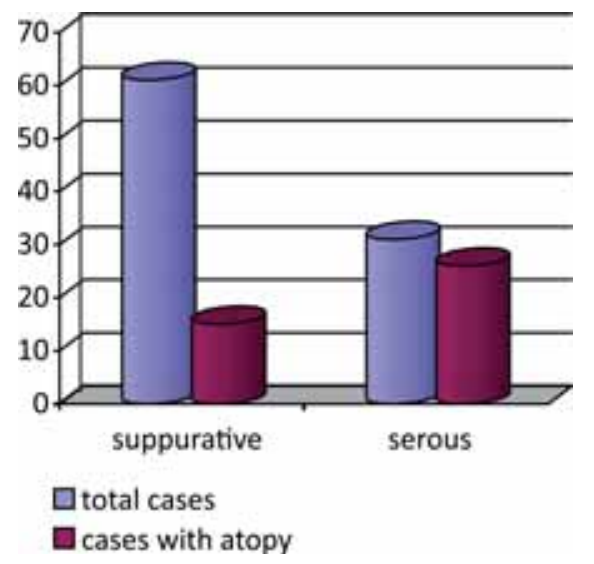

FIGURE 1. Distribution depending on otitis type and atopic features

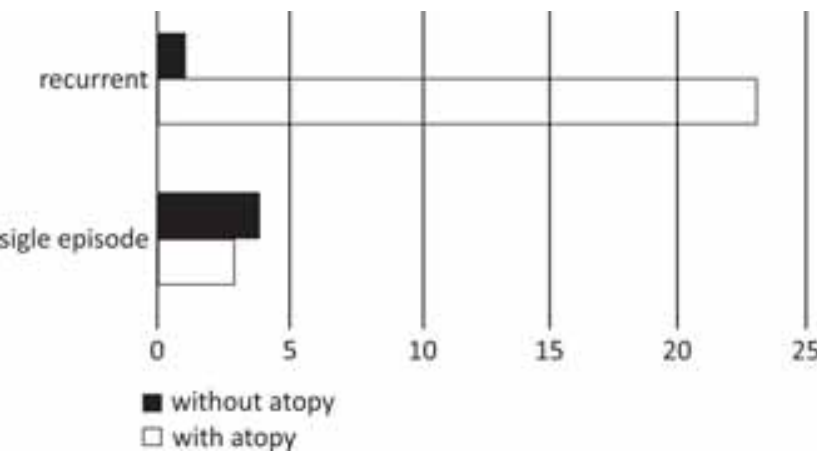

FIGURE 2. Distribution depending on numbers of episodes and atopic features

Regarding the allergic sensitization profile, it been noticed a relative high frequency of food allergy, particularly to cow milk protein (15 cases), followed by house dust sensitization. (Fig. 3)

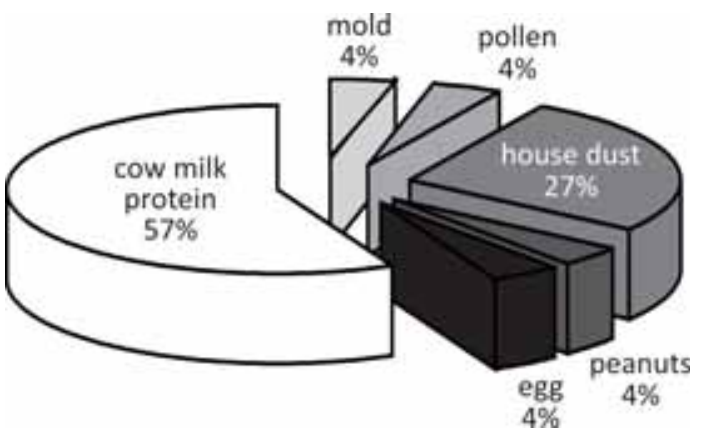

FIGURE 3. Allergic sensitivity profile

In most cases which presented cow milk allergy, the gastroesophageal reflux was present (10/15 cases).
The research on the association with the allergic diseases shows most frequent the concomitance with rhinitis or rhinoadenoiditis (54\%), with or without bronchial asthma. (Fig. 4)

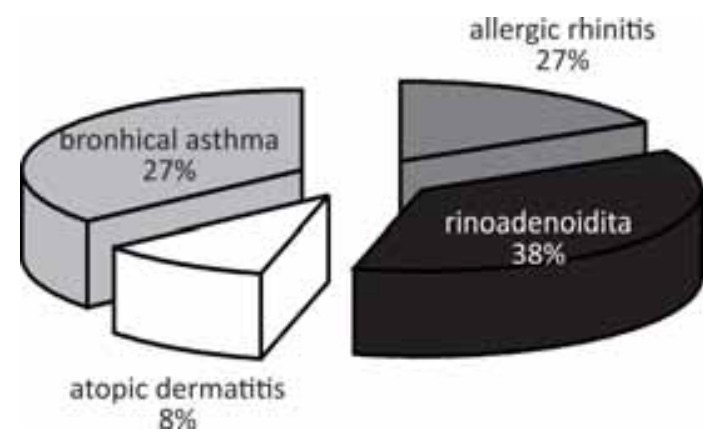

FIGURE 4. Associated atopic diseases

Suppurate otitis etiological spectrum was represented by S. pneumoniae, Staphilococcus aureus, Moraxella catarhallis and, more rarely, Haemophilus inluenzae. 4 patients presented allergic reaction to prescribed antibiotics (II generation cephalosporins) with major implication in the management of these cases.

\section{DISCUTION}

IgE mediated hypersensitivity relationship with recurrent serous otitis media has been demonstrated by numerous studies. Adenoidal tissue of nonallergic children does not contain or contain a few $\operatorname{IgE}+$ cells, in contrast with tissue of atopic persons, where, especially in the extrafolicular zones, it been uncounted an extremely large number of mast cells or macrophages IgE+. (1) Nasal endoscopy with the analysis of biopsies would play a major role in confirming the diagnosis and to prove the real association between otitis and atopy. (2) In the study group, the allergic pattern was performed by dosing the total serum IgE levels, most children associating rhinitis or rhinoadenoiditis.

Assessment of the role of allergy in the development of otitis media was carried out more in term of food allergy and the results are controversial. (3) A cohort study conducted on 262 children, underlines a correlation statistically significant between allergic rhinitis and otitis media with effusion, without significantly correlation with sensitization to pollen or dust. (4) In the study group, the most of the cases associate food allergy (especially cow milk protein allergy) followed by allergy to house dust.

Prevalence of gastroesophageal reflux disease in children with otitis media may be higher than 
that in studied population. (5) In our group, we observe a significant association for both suppurated and serous otitis, especially in those who associate cow milk protein allergy, elements often discussed in international studies. Immunoassays tests or esophageal ph-metry can be useful if it suspected an association between reflux and allergy (6), although only elimination diet and standardized challenge tests are certain diagnosis criteria. (7) The presence of pepsin or pepsinogen in ear exudate, in young infants, can be determinate by physiological reflux, the causal relationship between the two entities being not clearly elucidated. (8)

The emergence of otitis media with effusion in allergic children is not based only on the obstruction and the inflammation of Eustachian tube, but, mainly, the allergic inflammation of the respiratory epithelium including middle ear mucosa. The antihistaminic therapy and intranasal corticosteroid application association to standard therapy of otitis is

\section{REFERENCES}

1. Döner F., Yariktas M., Demirci M. The role of allergy in recurrentotitis media witheffusion J Invest Allergol Clin Immunol 2004; Vol. 14(4): 154-158

2. Marseglia G.L., Poddighe D., Caimmi D. et al. Role of adenoidsandadenoiditis in childrenwithallergyandotitis media Current Allergy and Asthma Reports 2009, Vol 9 (6): 460-464

3. Hurst DS The role of allergy in otitis media witheffusion. Otolaryngol Clin North Am. 2011 Jun; vol 44(3):637-654

4. Kreiner-Møller E., Chawes B.L., Caye-Thomasen P., Bønnelykke K., Bisgaard H. Allergic rhinitis is associated with otitis media with effusion: a birth cohort study, Clin Exp Allergy. 2012; 42(11):1615-1620

5. Chul K., Ho Y.L., Myung G.K., Sung H.B., Seung G.Y. Allergicdiseases in childrenwithotitis media with effusion International Journal of Pediatric Otorhinolaryngology Vol 77, (2)2013: 158-161

6. Yüksel F., Doğan M., Karataş D., Yüce S., Şentürk M., Külahli I. Gastroesophageal reflux disease in children with chronic otitis media witheffusion. J Craniofac Surg. 2013; vol 24(2):380-383 not supported entirely by specialized studies, being necessary to demonstrate the coexistence of these two entities. $(4,11)$ In the study group, after anamnesis suspicion or if the immunological markers of atopy were present, the specific and diet therapy was initiated, with long-term positive development, especially in serous recurrent otitis.

\section{CONCLUSION}

Children with otitis media, particularly those with serous forms, should be investigated to determine the association of atopy, the treatment of these cases being multidisciplinary: oto - rhino - laryngologist, allergologist and pediatrician. If the cow milk allergy is associated also, these patients should be investigate for gastresophageal reflux, eventually with pepsin dosage in otic secretion, in order to a targeted therapeutic management.

7. McCoul E.D., Goldstein N.A., Koliskor B., Weedon J., Jackson A., Goldsmith A.J. A prospective study of the effect of gastroesophageal reflux disease treatment on children with otitis media. Arch Otolaryngol Head Neck Surg. 2011; vol 137(1):35-41.

8. Gomaa M.A; Karim A.R., Elsherbeny Y.M. Role of immunoglobulin $E$ and gastro-esophageal reflux disease in the development of otitis media with effusion, Otolaryngologia Polska, Vol. 68 (3); 2014:119-123

9. Miura M.S., Mascaro M., Rosenfeld R.M. Association between otitis media and gastroesophageal reflux: a systematic review. Otolaryngol Head Neck Surg. 2012 146(3):345-352.

10. Luong A., Roland P.S. The link between allergic rhinitis and chronic otitis media with effusion in atopic patients, Otolaryngol Clin North Am. 2008; 41(2):311-23 